\title{
Prevalência e fatores associados a dependência funcional em idosos longevos
}

\author{
Prevalence and factors associated with functional dependence in the \\ oldest old
}

Emanuelly Casal Bortoluzzi , Marlene Doring², Marilene Rodrigues Portella², Gustavo Cavalcanti , Andréia Mascarelo², Marcos Paulo Delani

\section{Resumo \\ Objetivou-se avaliar a prevalência e os fatores associados a de- pendência funcional de idosos longevos residentes em muni- cípios de pequeno porte. Trata-se de um estudo transversal de base populacional com idosos de 80 anos e mais, recorte dos estudos sobre as condições de saúde de idosos dos municípios de Coxilha-RS e Estação-RS. Os dados foram coletados por meio de questionário adaptado da pesquisa Saúde, Bem-Estar e Envelhecimento (SABE). Participaram 77 idosos longevos (63,3\% mulheres). A prevalência de dependência funcional foi $26 \%\left(\mathrm{IC}_{95 \%} 16,8-36,4\right)$ e dentre as atividades básicas da vida diá- ria (ABVD) deitar e levantar da cama $(31,6 \%)$ e locomover-se $(31,2 \%)$ representaram a maior frequência de dependência. Na análise de regressão multivariada mostraram-se associadas à dependência funcional a autopercepção de saúde negativa $(\mathrm{RP}=$ 1,$\left.20 ; \mathrm{IC}_{95 \%} 1,05-1,37\right)$, não praticar atividade física $(\mathrm{RP}=1,20$; $\left.\mathrm{IC}_{95 \%} 1,05-1,36\right)$ e possuir três ou mais doenças crônicas $(\mathrm{RP}=$ 1,$\left.24 ; \mathrm{IC}_{95 \%} 1,08-1,41\right)$. Nesse sentido, observamos que os idosos longevos com a condição de dependência possuem como razão de prevalência fatores passíveis de prevenção, e sobretudo que essa condição é evitável ou possível de ser postergada.}

\section{Palavras-chave}

Idoso de 80 anos ou mais; Limitação física; Atividades cotidianas; Saúde do idoso; Cidades pequenas.

\begin{abstract}
This study aimed to assess the prevalence and factors associated with functional dependence of the oldest elderly people living in small towns. This is a cross-sectional population-based study with aged, 80 and over, cut out studies on the health conditions of the elderly in the municipalities of Coxilha-RS and Estação-RS. Data were collected through adapted questionnaire Health, Well-Being and aging (SABE). Study participants were 77 oldest elderly (63,3\% women). The prevalence of functional dependence was $26 \%\left(I C_{95 \%} 16,8-36,4\right)$ and among the basic activities of daily living $(A D L)$ bed and out of bed (31,6\%) and getting yourself (31,2\%) accounted for the frequency dependence. In multivariate regression analysis were associated with functional dependence negative self-rated health $\left(R P=1,20 ; I C_{95 \%} 1,05-1,36\right)$, not physical activity $\left(R P=1,19 ; I C_{95 \%} 1,05-1,36\right)$ and have three or more chronic diseases $\left(R P=1,24 ; I C_{95 \%} 1,08-1,41\right)$. In this regard, we note that the oldest old with the condition of dependency have as prevalence ratio preventable factors, and above all that this condition is preventable or can be postponed.
\end{abstract}

\section{Keywords}

Aged, 80 And Over; Disabled Persons; Activities of Daily Living; Health of the Elderly; Small Cities.

\section{Introdução}

O envelhecimento populacional é uma realidade mundial e a própria população idosa, em termos demográficos, está envelhecendo, pois registra-se um crescimento acelerado no segmento dos idosos longevos (idosos com idade igual ou superior a 80 anos). No Brasil os idosos representam cerca de $11 \%$ da população, dos quais $1,5 \%$ são longevos ${ }^{1}$.
A idade avançada é um fator determinante para o declínio da funcionalidade e o reconhecimento desta possibilita a identificação da necessidade de ajuda. Ainda, a capacidade funcional é um importante indicador de saúde que se refere à condição da pessoa idosa viver de forma autônoma e independente ${ }^{2}$. Nesse sentido, idosos longevos apresentam comportamentos sedentários com maior frequência e redução na prática de atividade física o que repercute negativamente sobre a saúde ${ }^{3}$.

Estudos indicam que fatores como baixo nível de 
atividade física, fadiga e dor podem ser preditores de dependência funcional, bem como os problemas físicos, a presença de múltiplas doenças crônicas e o uso excessivo de medicamentos pode comprometer a capacidade funcional e, consequentemente, interferem e reduzem a qualidade de vida dos idosos ${ }^{4,5}$.

A Organização Mundial da Saúde interpreta a capacidade funcional, como o resultado da interação entre o indivíduo e o ambiente considerando atributos relacionados à saúde ${ }^{6}$. A avaliação desta inclui as atividades básicas da vida diária (ABVD), as quais compreendem o autocuidado ${ }^{7}$. Logo, a dependência pode ser compreendida como a necessidade de atenção, apoio ou ajuda de outras pessoas para realizar as ABVD.

Diante do aumento no contingente de idosos longevos, num país como o Brasil, em que $45 \%$ dos municípios têm população inferior a dez mil habitantes ${ }^{8}$, o conhecimento da capacidade funcional dos idosos e fatores associados, se constitui em importante subsídio tanto para a gestão municipal na construção e implementação de políticas locais, bem como, para a equipe de saúde no estabelecimento das bases necessárias para um sistema de cuidado. A prevenção, a detecção e o controle precoce das doenças crônicas, a reversão ou redução dos declínios da funcionalidade, o gerenciamento de doenças crônicas avançadas e o incentivo e promoção de comportamentos que melhorem a capacidade ou compensem as perdas poderão contribuir positivamente para um envelhecimento saudável ${ }^{6}$. Assim, este estudo objetivou avaliar a prevalência e fatores associados a dependência funcional em idosos longevos de dois municípios de pequeno porte do norte do Rio Grande do Sul.

\section{Métodos}

Trata-se de um estudo transversal de base populacional, recorte das pesquisas "Condições de vida e saúde dos idosos no município de Coxilha-RS"9 e "Condições de vida e saúde dos idosos residentes em um município de pequeno porte no sul do Brasil” ${ }^{10}$ os quais foram realizados nos municípios de Coxilha/RS e Estação/RS, respectivamente. Ambos os estudos foram realizados no Programa de Pós-Graduação em Envelhecimento Humano da Universidade de Passo Fundo.

O município de Coxilha pertence a região Noroeste do RS, conta com 2.826 habitantes e Índice de Desenvolvimento Humano (IDH) alto $(0,706)$. Dentre os habitantes, foram selecionados todos os idosos do município $(\mathrm{n}=352)$, houve perda de $6 \%$ por recusa em participar da pesquisa ou devido ao participante não ter sido encontrado após três tentativas de visita, totalizando 331 idosos.

O município de Estação também é localizado na região noroeste do RS, possui IDH alto $(0,753)$ e 6.011 habitantes dos quais 992 eram idosos, aplicou-se a fórmula de populações finitas $(\mathrm{n}=400)$ e adicionou-se $10 \%$, considerando possíveis perdas, totalizando 420 idosos. Os quais foram selecionados, aleatoriamente, com base nos registros da Estratégia de Saúde da Família. Para tanto, os idosos foram listados por zonas de residência e sexo e, a seguir, foram selecionados por amostragem aleatória, mantendo as proporções estipuladas pela amostra.

A opção por agregar os dados dos dois municípios ocorreu pela necessidade de obter-se uma amostra maior de longevos. Isto foi possível pois os dois estudos utilizaram metodologias semelhantes ${ }^{9-10}$, e o teste de homogeneidade do qui-quadrado não mostrou diferenças estatisticamente significativa $(p>0,05)$ entre local de moradia e as variáveis sexo, faixa etária, morbidade e capacidade funcional.

Tendo em vista a grande variabilidade de métodos de investigação e de prevalência do desfecho nos diferentes estudos, para o cálculo de amostra, levou-se em 
consideração nível de confiança de $95 \%$, poder estatístico de $80 \%$, prevalência da doença no grupo exposto de $40 \%{ }^{11}$, razão não exposto-exposto $2: 1$, totalizando 81 idosos longevos.

Adotou-se como critério de inclusão possuir 80 anos ou mais e residir nos municípios do estudo há pelo menos seis meses. Dos 88 longevos avaliados foram excluídos 11 que apresentaram um escore $\leq 12$ pontos na versão modificada e validada do Mini Exame do Estado Mental ${ }^{12}$, totalizando 77 idosos longevos.

A coleta de dados foi realizada por meio de inquérito domiciliar, no período de 2010 e 2011, por profissionais e agentes de saúde previamente treinados, utilizando as seções A (informações pessoais), B (avaliação cognitiva), D (condições de saúde e hábitos de vida) e $\mathrm{E}$ (avaliação funcional) do questionário-base do estudo multicêntrico denominado SABE - Saúde, Bem estar e Envelhecimento, coordenado pela Organização Pan-Americana de Saúde para investigar condições de vida e saúde das pessoas idosas da América Latina e Caribe ${ }^{12}$.

Para a caracterização da amostra foram avaliadas as características sociodemográficas: faixa etária (80 a 84 anos e 85 anos e mais), sexo (feminino e masculino) cor (branco e preto/pardo), zona de moradia (urbana e rural), estado marital atual (divorciado/separado, viúvo e casado/amasiado), renda (até um salário mínimo, entre um e dois salários, de três a cinco salários e mais de cinco salários), e saber ler e escrever ( $\operatorname{sim} /$ não). Ainda, foram investigadas as variáveis atividade física autorreferida ( $\operatorname{sim} /$ não) dor no mínimo há três meses ( $\operatorname{sim} /$ não), multimorbidade, definida como presença de duas ou mais doenças crônicas $(\operatorname{sim} / \text { não })^{13}$ e número de doenças (nenhuma a duas doenças/três doenças ou mais). Para a variável atividade física, os idosos foram questionados se eles praticavam alguma atividade física no dia-a-dia (atividades domésticas, de trabalho e/ou de lazer) explicando e exemplificando para que pudessem compreender e classificar se praticavam ou não atividade física.

Quanto a variável desfecho, capacidade funcional para atividades básicas da vida diária, inclui as questões sobre alimentar-se, vestir-se, cuidar da aparência, deitar e levantar da cama, ir ao banheiro e locomover-se. Para cada questão as opções de resposta eram: sem dificuldade, pouca dificuldade, muita dificuldade, só consegue com ajuda e não consegue realizar. Para análise bivariada e multivariada os longevos foram categorizados em independentes (sem dificuldade/pouca dificuldade em uma ou mais atividades) e dependentes (muita dificuldade/ só realiza com ajuda/não consegue realizar uma ou mais atividades).

$\mathrm{Na}$ análise estatística empregou-se, para as variáveis numéricas as medidas de média e desvio padrão, e para as variáveis categóricas as frequências absolutas e relativas e intervalo de confiança de $95 \%$. Para a análise bivariada e multivariada utilizou-se a regressão de Poisson, com variância robusta, onde utilizou-se o procedimento stepwise backward, ou seja, no modelo de regressão foram incluídas as variáveis com valor de $\mathrm{p}<0,20$ na análise bivariada, retirando as variáveis uma a uma, de acordo com a perda de significância estatística $(p>0,05)$. Optou-se pela regressão de Poisson, com variância robusta, pois em estudos transversais, com prevalência do desfecho maior que $10 \%$, esta é a análise mais indicada, pois a regressão logística múltipla, superestima OR em estudos transversais com prevalência elevada (maior que $10 \%)^{14}$.

Todos os participantes das pesquisas assinaram o Termo de Consentimento Livre e Esclarecido. Ambas foram aprovadas pelo Comitê de Ética em Pesquisa da Universidade de Passo Fundo sob os pareceres n 148/2010 e 017/2011. 


\section{Resultados}

Participaram do estudo 77 sujeitos com 80 anos ou mais com idade mediana de 83 anos $\left(\mathrm{P}_{25}: 81-\mathrm{P}_{75}: 85\right)$, variando de 80 a 102 anos. Desses, a maioria eram do sexo feminino, brancos, viúvos, sabem ler e escrever, residem na zona urbana, vivem acompanhados, possuem renda entre 1 e 2 salários mínimos. Ainda, cerca de $65 \%$ referiram não praticam atividade física, quanto a presença de dor, essa foi relatada pela maioria dos longevos e a prevalência de multimorbidade foi 71,4\% (Tabela 1).

TABELA 1 - Características sociodemográficas, comportamentais e clínicas de idosos longevos dos municípios de Coxilha, 2010 e Estação, 2011, Rio Grande do Sul, Brasil.

\begin{tabular}{|c|c|c|}
\hline Variáveis & n (\%) & $I C_{95 \%}$ \\
\hline \multicolumn{3}{|l|}{ Faixa etária } \\
\hline 80 a 84 anos & $53(68,8)$ & $60,9-80,6$ \\
\hline 85 anos ou mais & $24(31,2)$ & $19,4-39,1$ \\
\hline \multicolumn{3}{|l|}{ Sexo } \\
\hline Masculino & $28(36,4)$ & $23,3-46,9$ \\
\hline Feminino & $49(63,6)$ & $53,1-76,7$ \\
\hline \multicolumn{3}{|l|}{ Cor } \\
\hline Branco & $66(85,7)$ & $77,8-93,6$ \\
\hline Preto/pardo & $11(14,3)$ & $6,4-22,1$ \\
\hline \multicolumn{3}{|l|}{ Zona de moradia } \\
\hline Urbana & $43(55,8)$ & $44,1-68,9$ \\
\hline Rural & $34(44,2)$ & $31,1-55,9$ \\
\hline \multicolumn{3}{|l|}{ Estado marital } \\
\hline Separado/solteiro & $3(3,9)$ & $0,0-9,1$ \\
\hline Viúvo & $43(55,8)$ & $44,1-63,7$ \\
\hline Casado/Amasiado & $31(40,3)$ & $29,9-53,2$ \\
\hline \multicolumn{3}{|l|}{ Renda } \\
\hline Até 1 SM & $29(38,2)$ & $27,6-47,4$ \\
\hline 1 a 2 SM & $32(42,1)$ & $28,9-54,0$ \\
\hline 3 a 5 SM & $12(15,8)$ & $7,9-25,1$ \\
\hline Acima de 5 SM & $3(3,9)$ & $0,0-10,6$ \\
\hline \multicolumn{3}{|l|}{ Sabe ler e escrever } \\
\hline Sim & $50(64,9)$ & $53,1-75,4$ \\
\hline Não & $27(35,1)$ & $24,6-49,9$ \\
\hline \multicolumn{3}{|c|}{ Dor continua ( $\geq 3$ meses) } \\
\hline Sim & $49(63,6)$ & $54,5-72,8$ \\
\hline Não & $28(36,4)$ & $27,2-45,5$ \\
\hline \multicolumn{3}{|l|}{ Atividade Física* } \\
\hline Sim & $27(35,1)$ & $24,6-48,2$ \\
\hline Não & $50(64,9)$ & $51,8-75,4$ \\
\hline \multicolumn{3}{|l|}{ Multimorbidade } \\
\hline Sim & $55(71,4)$ & $61,0-81,9$ \\
\hline Não & $22(28,6)$ & $18,1-39,0$ \\
\hline \multicolumn{3}{|c|}{ Autopercepção de saúde } \\
\hline Positiva & $32(42,1)$ & $31,5-51,5$ \\
\hline Negativa & $44(57,9)$ & $48,5-68,5$ \\
\hline \multicolumn{3}{|l|}{ Número de doenças } \\
\hline $0 a 2$ & $39(50,6)$ & $38,9-61,1$ \\
\hline 3 ou mais & $38(49,4)$ & $38,9-61,1$ \\
\hline
\end{tabular}

* Realiza no dia-a-dia atividades domésticas, de trabalho e/ou de lazer. 
A mediana de doenças crônicas foram $2\left(\mathrm{P}_{25}: 1-\mathrm{P}_{75}: 4\right)$, variando entre nenhuma a sete. Quanto a variável desfecho, a prevalência de independência funcional foi $74 \%$ ( $\left.\mathrm{IC}_{95 \%} 63,6-83,2\right)$, e dependência foi $26 \%\left(\mathrm{IC}_{95 \%} 16,8-36,4\right)$ e as atividades básicas realizadas com algum grau de dificuldade ou impossibilidade de realizar, foram com maior frequência, deitar e levantar da cama (31,6\%), locomover-se $(31,2 \%)$, vestir-se e banhar-se (28,6\% cada) (Tabela 2$)$.

TABELA 2 - Descrição das atividades básicas da vida diária autorreferidas pelos idosos longevos dos municípios de Coxilha, 2010 e Estação, 2011, Rio Grande do Sul, Brasil.

\begin{tabular}{|c|c|c|c|c|c|c|c|c|c|c|}
\hline \multirow{3}{*}{ Variáveis } & \multicolumn{4}{|c|}{ Independentes } & \multicolumn{6}{|c|}{ Dependentes } \\
\hline & \multicolumn{2}{|c|}{ Sem dificuldade } & \multicolumn{2}{|c|}{ Pouca dificuldade } & \multicolumn{2}{|c|}{ Muita dificuldade } & \multicolumn{2}{|c|}{ Só com ajuda } & \multicolumn{2}{|c|}{ Não consegue } \\
\hline & $n(\%)$ & $I C_{95 \%}$ & $n(\%)$ & $I C_{95 \%}$ & $n(\%)$ & $I C_{95 \%}$ & $n(\%)$ & $I C_{95 \%}$ & $n(\%)$ & $I C_{95 \%}$ \\
\hline Alimentar-se & $67(87,0)$ & $81,7-94,8$ & $5(6,5)$ & $1,3-13,1$ & $1(1,3)$ & $0,0-3,9$ & $2(2,6)$ & $0,0-6,5$ & $2(2,6)$ & $0,0-5,2$ \\
\hline Banhar-se & $55(71,4)$ & $64,9-80,7$ & $6(7,8)$ & $1,3-15,6$ & $2(2,6)$ & $0,0-7,8$ & $11(14,3)$ & $3,9-22,2$ & $3(3,9)$ & $0,0-9,1$ \\
\hline Vestir-se & $55(71,4)$ & $59,7-80,6$ & $11(14,3)$ & $7,7-23,4$ & $2(2,6)$ & $0,0-7,8$ & $5(6,5)$ & $2,5-11,8$ & $4(5,2)$ & $1,2-13,0$ \\
\hline Cuidar da aparência & $58(75,3)$ & $67,5-83,3$ & $9(11,7)$ & $5,1-18,2$ & $2(2,6)$ & $0,0-6,5$ & $5(6,5)$ & $2,5-13,0$ & $3(3,9)$ & $0,0-7,8$ \\
\hline Deitar/levantar da cama & $52(68,4)$ & $57,7-80,3$ & $8(10,5)$ & $5,2-18,5$ & $10(13,2)$ & $7,8-22,4$ & $4(5,3)$ & $0,0-10,5$ & $2(2,6)$ & $0,0-6,6$ \\
\hline Ir ao banheiro & $61(79,2)$ & $68,8-85,9$ & $6(7,8)$ & $1,3-15,7$ & $5(6,5)$ & $2,5-13,0$ & $3(3,9)$ & $0,0-7,9$ & $2(2,6)$ & $0,0-5,2$ \\
\hline Locomover-se & $53(68,8)$ & $59,7-79,3$ & $9(11,7)$ & $5,1-18,2$ & $11(14,3)$ & $7,6-22,1$ & $1(1,3)$ & $0,0-5,2$ & $3(3,9)$ & $0,0-10,4$ \\
\hline
\end{tabular}

Quanto a ser independente nas ABVD, as maiores proporções são referidas por longevos do sexo masculino, da cor branca, residentes da zona rural, que moram sozinhos, casados, com renda entre três e cinco salários e que sabem ler e escrever. Ainda, são independentes a maioria dos longevos sem dor crônica, que nunca fumaram, não possuem multimorbidade e tem autopercepção de saúde positiva. $\mathrm{Na}$ análise bivariada mostraram-se associadas a dependência funcional as variáveis dor, atividade física autorreferida, multimorbidade, autopercepção de saúde e número de doenças crônicas ( $p>0,05)$ (Tabela 3$)$.

TABELA 3 - Análise bivariada entre capacidade funcional e variáveis sociodemográficas, comportamentais e clínicas dos idosos longevos dos municípios de Coxilha, 2010 e Estação, 2011, Rio Grande do Sul, Brasil.

\begin{tabular}{|c|c|c|c|}
\hline \multirow{2}{*}{ Variáveis } & Dependente & \multirow{2}{*}{$\mathrm{RP}_{\text {bruta }} \mid \mathrm{C}_{95 \%}$} & \multirow{2}{*}{$p$} \\
\hline & n (\%) & & \\
\hline \multicolumn{4}{|l|}{ Faixa etária } \\
\hline 80 a 84 anos & $12(22,6)$ & 1 & \\
\hline 85 anos ou mais & $8(33,3)$ & $1,09(0,92-1,29)$ & 0,331 \\
\hline \multicolumn{4}{|l|}{ Sexo } \\
\hline Masculino & $6(21,4)$ & 1 & \\
\hline Feminino & $14(28,6)$ & $1,06(0,90-1,24)$ & 0,482 \\
\hline \multicolumn{4}{|l|}{ Cor } \\
\hline Branco & $16(24,2)$ & 1 & \\
\hline Não branco & $4(36,4)$ & $1,09(0,88-1,37)$ & 0,416 \\
\hline \multicolumn{4}{|l|}{ Zona de moradia } \\
\hline Urbana & $14(32,6)$ & 1 & \\
\hline Rural & $6(17,6)$ & $0,89(0,76-1,03)$ & 0,123 \\
\hline \multicolumn{4}{|l|}{ Estado marital } \\
\hline Casado/Amasiado & $5(16,1)$ & 1 & \\
\hline Viúvo & $14(32,6)$ & $1,14(0,98-1,33)$ & 0,091 \\
\hline
\end{tabular}


... contina

\begin{tabular}{|c|c|c|c|}
\hline Separado/solteiro & $1(33,3)$ & $1,14(0,76-1,74)$ & 0,514 \\
\hline \multicolumn{4}{|l|}{ Renda } \\
\hline Até 1 salário & $7(24,1)$ & 1 & \\
\hline 1 a 2 salários & $8(25,0)$ & $1,01(0,85-1,19)$ & 0,938 \\
\hline 3 a 5 salários & $3(25,0)$ & $1,01(0,79-1,27)$ & 0,954 \\
\hline Acima de 5 salários & $2(66,7)$ & $1,34(0,95-1,89)$ & 0,093 \\
\hline \multicolumn{4}{|l|}{ Sabe ler e escrever } \\
\hline Sim & $13(26,0)$ & 1 & \\
\hline Não & $7(25,9)$ & $0,99(0,85-1,18)$ & 0,994 \\
\hline \multicolumn{4}{|l|}{ Dor continua } \\
\hline Não & $3(10,7)$ & 1 & \\
\hline Sim & $17(34,7)$ & $1,22(1,05-1,40)$ & 0,007 \\
\hline \multicolumn{4}{|l|}{ Atividade Física* } \\
\hline Sim & $1(3,7)$ & 1 & \\
\hline Não & $19(38,0)$ & $1,33(1,18-1,49)$ & $<0,001$ \\
\hline \multicolumn{4}{|l|}{ Multimorbidade } \\
\hline Não & - & 1 & \\
\hline Sim & $20(36,4)$ & $1,36(1,24-1,49)$ & $<0,001$ \\
\hline \multicolumn{4}{|c|}{ Autopercepção de saúde } \\
\hline Positiva & $2(6,3)$ & 1 & \\
\hline Negativa & $17(38,6)$ & $1,30(1,14-1,49)$ & $<0,001$ \\
\hline \multicolumn{4}{|l|}{ Número de doenças } \\
\hline 0 a 2 & $3(7,7)$ & 1 & \\
\hline 3 ou mais & $17(44,7)$ & $1,34(1,17-1,54)$ & $<0,001$ \\
\hline
\end{tabular}

* Realiza no dia-a-dia atividades domésticas, de trabalho e/ou de lazer.

No modelo múltiplo, foram incluídas as variáveis que apresentaram, no mínimo em uma das categorias $\mathrm{p}<0,20$, que compreendeu as variáveis zona de moradia, estado civil, renda, dor, atividade física, multimorbidade, autopercepção de saúde e número de doenças crônicas. As quais foram retiradas do modelo uma a uma de acordo com a perda de significância estatística. No modelo final mostraram-se associadas a dependência para ABVD a autopercepção de saúde negativa $\left(\mathrm{RP}=1,20 ; \mathrm{IC}_{95 \%} 1,05-1,37\right)$, não praticar atividade física $\left(\mathrm{RP}=1,20 ; \mathrm{IC}_{95 \%} 1,05-1,36\right) \mathrm{e}$ possuir três ou mais doenças crônicas $\left(\mathrm{RP}=1,24 ; \mathrm{IC}_{95 \%} 1,08-1,41\right)$ (Tabela 4).

TABELA 4 - Modelo múltiplo final dos fatores associados a dependência funcional para ABVD dos idosos longevos dos municípios de Coxilha, 2010 e Estação, 2011, Rio Grande do Sul, Brasil.

\begin{tabular}{lcc}
\hline Variáveis & $R_{\text {ajustada* }} \mathcal{C}_{95 \%}$ & $P$ \\
\hline Autopercepção de saúde & 1 & \\
\hline Positiva & $1,20(1,05-1,37)$ & 0,007 \\
\hline Negativa & 1 & 0,007 \\
\hline Atividade física** & $1,20(1,05-1,36)$ & \\
\hline Sim & 1 & 0,002 \\
\hline Não & $1,24(1,08-1,41)$ & \\
\hline Número de doenças & & \\
\hline 3 a 2 & ou mais & \\
\hline
\end{tabular}

* RP ajustada por zona de moradia, renda, estado civil, dor contínua e multimorbidade. ${ }^{* *}$ Realiza no dia-a-dia atividades domésticas, de trabalho e/ou de lazer. 


\section{Discussão}

Ao avaliar a capacidade funcional de idosos longevos residentes em municípios de pequeno porte, encontrou-se associação com as variáveis autopercepção de saúde, atividade física e número de doenças crônicas. As quais demonstraram que a razão de prevalência de dependência funcional foi associada com possuir três ou mais doenças, autopercepção negativa de saúde e não praticar atividade física.

O estudo de Nogueira et al. ${ }^{2}$ avaliou a capacidade funcional de idosos longevos em São Geraldo MG/BR, no qual possuir mais de 85 anos e ser mulher mostraram-se associadas a dependência, resultado não encontrado no presente estudo. Fato que vem de encontro a conclusão dos mesmos autores, que recomendam cuidado quanto a generalização dos resultados, pois a capacidade funcional é influenciada por complexa rede multifatorial, e esses fatores podem diferir entre os locais, reafirmando a relevância do presente estudo.

Pode-se considerar como limitação deste estudo o tamanho reduzido da amostra, entretanto, esta é uma característica de estudos envolvendo longevos, visto que, este segmento apresenta pequena proporção na população em geral (cerca de $1,2 \%$ no Brasil ${ }^{1}$ ), principalmente quando se refere a municípios com menos de 10.000 habitantes. Outra limitação a ser considerada é a escala de atividades básicas de vida diária ser baseada no autorrelato. Porém, esta metodologia é amplamente utilizada e aceita em estudos epidemiológicos ${ }^{15-16}$. Nesse Contexto, uma revisão sistemática com metanálise que avaliou a capacidade funcional de idosos brasileiros, encontrou entre os estudos somente $8,7 \%$ objetivando os idosos longevos e todos os estudos eram baseados em informações autorreferidas para a capacidade funcional ${ }^{17}$.

Ao analisar os dados sociodemográficos, ressalta-se a feminilização da população estudada, em concordância com a literatura ${ }^{18-19}$, que pode ser explicada pelas diferentes características de mortalidade entre os $\operatorname{sexos}^{20}$. Ainda, a maioria mora acompanhada (o), são viúvas(os), sabem ler/escrever e tem renda de um a dois salários mínimos. Perfil semelhante ao encontrado em um estudo realizado, em uma capital brasileira, com longevos usuários de uma Unidade Básica de Saúde ${ }^{21}$. Essa realidade, mulheres, viúvas e de baixa renda, mostra-se como uma barreira adicional ao cuidado desses longevos na presença de dependência funcional ${ }^{22}$.

Observa-se que $74 \%$ dos idosos longevos são independentes para realizar suas atividades básicas, resultado semelhante a Nogueira et al., ${ }^{2}$ que encontraram $71 \%$ de longevos com capacidade funcional muito boa/boa. Dentre as atividades analisadas, as que foram citadas com maior frequência como realizadas com muita dificuldade ou impossibilidade de realização foram, deitar/levantar da cama e banhar-se, seguidas por, locomover-se e vestir-se. Essas atividades, exigem dentre outras capacidades físicas, a mobilidade articular e massa muscular, nesse sentido, o processo de envelhecimento gera uma diminuição da massa muscular entre os 50 a 80 anos, de cerca de 35\%, principalmente nos membros inferiores, mas esse declínio por si só, não é o responsável pela dependência funcional ${ }^{23}$.

Assim, quando o processo de envelhecimento é potencializado pela presença de doenças crônicas e não realização de atividades físicas, pode chegar a tal limiar de comprometimento funcional ${ }^{23}$. Da mesma forma, a mobilidade articular também pode ter seu declínio diminuído quando há a realização de exercícios de resistência e flexibilidade ${ }^{24}$. Nesse sentido, não praticar atividade física mostrou-se associada a dependência, corroborando ao estudo de Ribeiro et al., ${ }^{25}$ no qual a pratica de atividade física contribui para a independência funcional.

Destaca-se ainda que, devido as alterações decorrentes do processo de envelhe- 
cimento ocorre a diminuição da tolerância ao exercício ${ }^{25}$. Porém, mesmo os idosos dependentes necessitam da prática de atividade física, a qual precisa ser adequada e adaptada para a condição de saúde do indivíduo ${ }^{26}$.

Quanto as doenças crônicas, a multimorbidade perdeu a significância no modelo múltiplo, porém possuir três ou mais doenças crônicas manteve-se como razão de prevalência para a dependência funcional. Este resultado contrapõe-se ao estudo realizado em Curitiba-PR, o qual não encontrou associação entre o número de doenças crônicas e dependência ${ }^{25}$. O que pode ser explicado pelas diferentes características culturais, socioeconômicas e estilo de vida, tais como, menor exposição a poluição, congestionamentos, violência e menor custo de vida, encontradas em municípios de pequeno em comparação a municípios de grande porte. Aliado a isso, deve-se considerar os diferentes métodos de avaliação da capacidade funcional utilizada nos dois estudos.

A autopercepção de saúde é um indicador multidimensional, que agrega fatores sociodemográficos, ambientais, sociais, culturais e clínicos ${ }^{27}$ e, portanto, é considerado um método eficaz e confiável para mensurar aspectos de saúde da população ${ }^{28}$. Nesse sentido, a autopercepção de saúde negativa mostrou-se associada a dependência para capacidade funcional, o que reforça a sua validade como um indicador que demonstra as reais condições de saúde dos idosos.

Com relação a assistência à saúde, Arnau et al., ${ }^{29}$ encontraram como principal fator de risco para diminuição da capacidade funcional a internação hospitalar. Ainda, estudo realizado na Suécia, aponta que cerca de 40\% dos idosos longevos internados poderiam receber os cuidados necessários fora do hospital. Portanto, os mesmos autores afirmam que idosos com múltiplas doenças necessitam de cuidados eficientes em saúde, o que não significa hospitalização, tendo em vista que evitar internações desnecessárias diminui o custo para a sociedade e principalmente o risco para o idoso ${ }^{30}$.

Os achados desse estudo demonstram que cerca de um terço dos idosos longevos residentes em municípios de pequeno porte possuem dependência funcional, e apresentam como fatores associados a dependência a autopercepção de saúde negativa, não praticar atividade física e possuir três ou mais doenças crônicas. Nesse sentido, a condição de dependência funcional, exige um cuidado diferenciado pelos familiares e serviço de saúde, haja vista que, ao possuir acesso a esses cuidados a condição de dependência pode diminuir ou apresentar-se com menor sofrimento, tristeza e isolamento.

Portanto é preciso que a sociedade, familiares e os serviços de saúde, atentem para as reais necessidades de cuidados dos idosos e tratem a dependência funcional dos idosos com 80 anos e mais como um processo evitável ou possível de retardar e não como um processo natural, para que possam oferecer o cuidado adequado a esses longevos e avaliação ampla de sua condição. Tendo em vista, que a longevidade com saúde é uma herança das escolhas que o indivíduo realiza ou é submetido, cabe a sociedade como um todo, agir em prol da promoção da saúde e prevenção das doenças, para que seja possível viver por mais tempo livre de incapacidades e com melhor qualidade de vida.

\section{Contribuiç̧ão dos autores}

Bortoluzzi EC. participou da concepção do artigo, análise dos dados, revisão de literatura e redação do manuscrito. Doring M. participou da análise dos dados, redação e revisão crítica do manuscrito. Portella MR. participou da revisão de literatura, redação e revisão crítica do manuscrito. Cavalcanti G. participou da con- 
cepção do artigo, análise dos dados e revisão de literatura. Mascarelo A. participou da coleta de dados, concepção e revisão do manuscrito. Delani MP. participou da coleta de dados, concepção e revisão do manuscrito.

\section{Referências}

1. Instituto Brasileiro de Geografia e Estatística. Projeções e estimativas da população do Brasil e das Unidades da Federação [online]; 2013. [citado 2016 ago 18]. Disponível em: http://goo.gl/pb23XR.

2. Nogueira SL, Ribeiro RCL, Rosado LEFP, Franceschini SCC, Ribeiro AQ, Pereira ET. Fatores determinantes da capacidade funcional em idosos longevos. Rev Bras Fisioter 2010; 14(4): 322-9.

3. Boscatto EC, Duarte MFS; Barbosa AR. Nível de atividade física e variáveis associadas em idosos longevos de Antônio Carlos, SC. Rev Bras Ativ Fís e Saúde 2012; 17(2): 132-136.

4. Avlund K. Fatigue in older adults: an early indicator of the aging process? Aging clinical and experimental research 2010; 22(2): 100-115.

5. Huang, WW, Perera S, VanSwearingen J, Studenski S. Performance measures predict onset of activity of daily living difficulty in community-dwelling older adults. J Am Geriatr Soc 2010; 58(5): 844-852.

6. Organização Mundial de Saúde. Relatório Mundial de Envelhecimento e Saúde. Genebra: Oragnização Mundial de Saúde; 2015.

7. Del Duca GF, Silva MC, Hallal PC. Incapacidade funcional para atividades básicas e instrumentais da vida diária em idosos. Rev Saúde Pública 2009; 43(5): 796-805.

8. Instituto Brasileiro de Geografia e Estatística. Estimativas da população dos municípios brasileiros com data de referência em $1^{\circ}$ de julho de 2014. 2015. Disponível em: http:// www.ibge.gov.br/home/presidencia/noticias/pdf/analise_estimativas_2014.pdf.

9. Mascarelo A. Condições de vida e saúde dos idosos no município de Coxilha-RS. Dissertação [Mestrado em Envelhecimento Humano] - Faculdade de Educação Física e Fisioterapia da Universidade de Passo Fundo, Passo Fundo - RS; 2012.

10. Delani MP. Condições de vida e saúde dos idosos residentes em um município de pequeno porte no sul do Brasil. Dissertação [Mestrado em Envelhecimento Humano] - Faculdade de Educação Física e Fisioterapia da Universidade de Passo Fundo, Passo Fundo - RS; 2012.

11. Aires M, Paskulin LMG, Morais EP. Capacidade funcional de idosos mais velhos: estudo comparativo em três regiões do Rio Grande do Sul. Rev Latino-Am Enfermagem 2010; 18(1): 11-17.

12. Lebrão ML, Laurenti R. Saúde, bem-estar e envelhecimento: o estudo SABE no Município de São Paulo. Rev bras epidemiol 2005; 8(2): 127-41.

13. Batista SR. A complexidade da multimorbidade. J Manag Prim Health Care 2014; 5(1): $125-26$.

14. Coutinho LMS, Scazufca M, Menezes PR. Métodos para estimar razão de prevalência em estudos de corte transversal. Rev Saúde Pública 2008; 42(6): 992-98.

15. Fialho CB, Lima-Costa MF, Giacomin KC, Loyola Filho AI. Capacidade funcional e uso de serviços de saúde por idosos da região metropolitana de Belo Horizonte, Minas Gerais, Brasil: um estudo de base populacional. Cad Saúde Pública 2014; 30(3): 599-610.

16. Lima AP, Lini EV, Tomicki C, Dellani M, Portella M, Doring M. Fatores associados à atividade física em idosos de Estação, Rio Grande do Sul: estudo de base populacional. Rev Bras Ativ Fís e Saúde 2015; 20(6): 618-625.

17. Campos ACV, Almeida MHM, Campos GV, Bogutchi TF. Prevalência de incapacidade funcional por gênero em idosos brasileiros: uma revisão sistemática com metanálise. Rev. Bras. Geriatr. Gerontol. 2016;19(3): 545-559.

18. Wandera SO, Kwagala B, Ntozi J. Prevalence and risk factors for self-reported noncommunicable diseases among older Ugandans: a cross-sectional study. Glob Health Action 2015; 8.

19. Chen W, Fang Y, Mao F, Hao S, Chen J, Yuan M, et al. Assessment of disability among the elderly in Xiamen of China: a representative sample survey of 14,292 older adults. PloS one $2015 ; 10(6)$ : e0131014. 
20. Porciúncula RCR, Carvalho EF, Barreto KML, Leite VMM. Perfil socioepidemiológico e autonomia de longevos em Recife-PE, Nordeste do Brasil. Rev Bras Geriat Gerontol 2014; 17(2): 315-25.

21. Lenardt MH, Carneiro NHK. Associação entre as características sociodemográficas e a capacidade funcional de idosos longevos da comunidade. Cogitare Enfermagem 2013; 18(1): 13-20.

22. Minayo MCS. Aumento acelerado da expectativa de vida e o desafio de cuidar das pessoas idosas dependentes. Investigaciones Andina 2015;17(31): 1273-78.

23. Valente M. Sarcopenia. In: Freitas, E.V.; Py, L. (Edit.) Tratado de Geriatria e Gerontologia. 3th Ed. Rio de Janeiro: Guanabara Koogan; 2011.

24. Ribeiro RG, Domingues DO, Silva VA. Treinamento de flexibilidade e sua relação com as atividades da vida diária no envelhecimento: um estudo de revisão. Rev Atenção à Saúde 2010; 6(17): 86-91.

25. Ribeiro DKMN, Lenardt MH, Michel T, Setoguchi LS, Grden CRB, Oliveira ES. Fatores contributivos para a independência funcional de idosos longevos. Rev Esc Enferm USP 2015; 49(1): 89-96.

26. Organização Mundial de Saúde. Global recommendations on physical activity for health. Geneva: Organização Mundial de saúde; 2010.

27. Nunes APN, Barreto SM, Gonçalves LG. Relações sociais e autopercepção da saúde: Projeto Envelhecimento e Saúde. Rev. bras. Epidemiol 2012; 15(2): 415-28.

28. Borges AM, Santos G, Kummer JA, Fior L, Molin VD, Wibelinger LM. Autopercepção de saúde em idosos residentes em um município do interior do Rio Grande do Sul. Rev. Bras. Geriatr. Gerontol. 2014; 17(1): 79-86.

29. Myredal A, Mauritzon I, Blom M, Ivarsson K. Many people over 80 years had been able to get care outside the hospital. Lakartidningen 2016; 2(1):113-120.

30. Arnau A, Espaulella J, Serrarols M, Canudas J, Formiga F, Ferrer M. Risk factors for functional decline in a population aged 75 years and older without total dependence: A one-year follow-up. Arch Gerontol Geriatr. 2016; 65: 239-247.

Endereço para

correspondência

Emanuelly Casal Bortoluzzi

manu_casal@hotmail.com
Rua Dona Verônica, n69, Bairro Quiri,

Ronda Alta/RS, CEP: 99670-000

Tel.: 54-99560403,
Recebido

Revisado

Aprovado
26/09/2016 $28 / 11 / 2016$ 20/12/2016 